\title{
Long Noncoding RNA ENST00000581794.1 in Peripher- al Blood Mononuclear Cells Acts as a Novel Biomarker in Patients with Acute Myocardial Infarction
}

\begin{abstract}
Z. XUE-HE, QIAN ZHAO, NING SONG ${ }^{1}$, JUN-YI LUO'1, C. QING-JIE ${ }^{1}$, A. CHENC², MUNIRE TUXUN, G. AIHEMAITI AND XIAO-MEI LI ${ }^{*}$

State Key Laboratory of Pathogenesis, Prevention and Treatment of High Incidence Diseases in Central Asia, Department of Cardiology, ${ }^{1}$ Xinjiang Key Laboratory of Cardiovascular Disease Research, Clinical Medical Research Institute of First Affiliated Hospital of Xinjiang Medical University, Urumqi, China, ${ }^{2}$ Beijing University of Chinese Medicine, Bei San Huan East Road, Beijing, China
\end{abstract}

Xue-He et al.: LncRNA ENST00000581794.1 a novel biomarker in AMI

\begin{abstract}
The aim of this investigation is to investigate the long noncoding ribonucleic acid and mRNA expression profiles of acute myocardial infarction patients and matched healthy controls particularly the higherranked long noncoding ribonucleic acid ENST00000581794.1 in co-expression. This study group found that ENST00000581794.1 was up-regulated in acute myocardial infarction patients in pre-experiment. It is suggested that ENST00000581794.1 may be a potential biomarker of acute myocardial infarction. Fifty eight patients with acute myocardial infarction and 58 healthy controls were included in this study from September 2017 to February 2018. Venous blood samples were collected from patients within $6 \mathrm{~h}$ from the onset of chest pain and RT-qPCR measured the expression levels of long noncoding ribonucleic acid in peripheral blood mononuclear cells. All patients were followed for $12 \pm 1 \mathrm{mo}$. The expression levels of ENST00000581794.1 were significantly different between the two groups. The receiver operating characteristic curve showed that long noncoding ribonucleic acid ENST00000581794.1 (area under the curve $=0.744,95 \%$ confidence interval: $0.61 \sim 0.88, p=0.004$ ) had a predictive effect on major adverse cardiovascular events. Survival curve analysis demonstrated that the incidence of major adverse cardiovascular events became significantly higher in patients with high expression of ENST00000581794.1 as the observation time increased. Multivariate regression analysis indicated that ENST00000581794.1 was an independent predictor of major adverse cardiovascular events in patients with acute myocardial infarction (hazard ratio: 1.17, $95 \%$ confidence interval: 1.00 1.37, $p=0.048$ ). Highly expressed ENST00000581794.1 in peripheral blood mononuclear cells may be used as a biomarker for diagnosis and prognosis of acute myocardial infarction.
\end{abstract}

Key words: IncRNA, acute myocardial infarction, major adverse cardiovascular events, biomarker, survival curve analysis, RT-qPCR

Acute myocardial infarction (AMI) is one of the most serious cardiovascular diseases seen in the clinic with high morbidity and mortality ${ }^{[1]}$. The condition is usually caused by the activation of platelet aggregation at ruptured or eroded atherosclerotic plaque sites. The diagnosis of AMI depends mainly on chest pain symptoms, electrocardiogram (ECG) results, and the presence of myocardial enzymes ${ }^{[2]}$. The early, rapid, and correct diagnosis of AMI has been a focus of research, as it can prevent AMI progression, improve recovery, survival rates and outcomes. Cardiac troponin I (cTnI) and creatine kinase-MB isoenzyme (CK-MB) are the biomarkers most commonly used in clinical practice to diagnose AMI. However, in addition to AMI, several other conditions may result in elevated levels of cTnI and CK-MB. Therefore, it is important to find sensitive and specific biomarkers for early MI. An ideal biomarker for AMI also requires good accessibility and predictability ${ }^{[3]}$.

Long noncoding RNA (lncRNA) is defined as a nonprotein-coding transcript longer than 200 nucleotides $^{[4]}$. lncRNA originally was thought to be a by-product of RNA polymerase II transcription and have no biological

*Address for correspondence

E-mail: romancia@126.com 
function. However, as research has progressed, more and more evidence has indicated that IncRNA is a key component of gene regulatory networks. Also, a large number of studies have highlighted the important role that IncRNA plays in various biological processes, including $\mathrm{X}$-chromosome silencing, genomic imprinting, chromatin modification, transcriptional activation, transcriptional interference, and nuclear transport. lncRNA is involved in the regulation of individual growth, differentiation, cell proliferation, apoptosis and other life activities ${ }^{[5,6]}$. Its abnormal expression is associated with a variety of diseases.

Recent studies have found that lncRNA is closely related to the occurrence of cardiovascular disease ${ }^{[7]}$. Large-scale association analysis showed that singlenucleotide polymorphism locus variation in lncRNAMIAT exon 5, which is associated with MI-related transcription, is closely related to MI susceptibility ${ }^{[8]}$. Subsequent clinical trials have also found a correlation between lncRNAs such as ANRIL ${ }^{[9]}$, UCA1 ${ }^{[10]}$ and LIPCAR $^{[11]}$ and the onset of AMI. Studies have shown that circulating lncRNAs are usually present as secondary structures and are relatively stable, which helps them to be used as biomarkers in body fluids such as plasma, serum and urine. Circulating RNA in body fluids is expected to be a promising biomarker for noninvasive diagnostic applications. However, the value of lncRNA for predicting the prognosis of MI has not been systematically studied.

In this investigation gene expression profiles in AMI and matched control group were screened to select the higher-ranked lncRNA ENST00000581794.1 from the co-expressed genes. After designing primers, a small number of primary experiments were performed. The result showed the level was significantly higher than the control group. This study aimed to expand the sample size further to verify the diagnostic value of ENST00000581794.1 for AMI in peripheral blood mononuclear cells (PBMCs) and clarify the impact of ENST00000581794.1 on the prognosis of AMI patients through follow-up along with assessment of the potential of ENST00000581794.1 as a novel biomarker for diagnosis and prognosis of AMI.

\section{MATERIALS AND METHODS}

From September 2017 to February 2018, 58 patients diagnosed with AMI associated with typical chest pain were admitted to the First Affiliated Hospital of Xinjiang Medical University. AMI was diagnosed based on the combination of several parameters, ischemic symptom plus increased $\mathrm{cTnI}$ and $\mathrm{CK}-\mathrm{MB}$, as well as a pathological Q wave. ST-segment elevation or depression was defined by the European Society of Cardiology/American College of Cardiology ${ }^{[12]}$. The gender and age-matched control subjects were recruited from the same hospital during the same period; the result of coronary angiography with no obvious stenosis. Before registration, data on age, height, weight, smoking history and cardiovascular risk factors were collected. A baseline ECG was recorded for all patients. All patients who were diagnosed with AMI received echocardiography and immediate percutaneous coronary intervention (PCI) as soon as possible. Exclusion criteria for the study included heart failure (HF) primarily due to severe valve disease and dilated cardiomyopathy, combined acute and chronic infection, serious liver and kidney dysfunction, malignancies and immune system diseases and cardiac complications affecting prognosis. All AMI patients received standard medical treatment, including $300 \mathrm{mg}$ of aspirin and a $300 \mathrm{mg}$ loading dose of clopidogrel at admission, and $70 \mathrm{U} / \mathrm{kg}$ of standard intravenous heparin before the PCI. All PCI procedures were performed by experienced interventional cardiologists using a radial artery approach and drug-eluting stents. After that, all patients received dual antiplatelet therapy, $100 \mathrm{mg}$ aspirin and $75 \mathrm{mg}$ of clopidogrel daily for at least one year. Other cardiac medications were given at the discretion of the attending physician. Written consents were obtained from all subjects, and the ethics committee of the Xinjiang Medical University approved the study protocol.

\section{Sample collection and detection of myocardial enzymes:}

Peripheral blood samples anticoagulated by ethylenediaminetetraacetic acid (EDTA) were collected from patients after hospital admission, within $6 \mathrm{~h}$ from the onset of chest pain. The remaining participants had fasting venous blood collected on the morning of admission. Specimens were placed in heparin anticoagulant tubes and centrifuged at $3000 \mathrm{rpm}$ for 10 min to separate the plasma. Human PBMCs were isolated using Lymphoprep (Axis-Shield, Norway) and then carefully transferred into an RNase-free tube (AxyGen, USA) for extraction of RNA. White blood cell count (WBC), total cholesterol (TC), triglyceride (TG), low- or high-density lipoprotein-cholesterol (LDL-C, HDL-C), creatinine, cardiac troponin I (cTnI, Roche Diagnostics, Indianapolis, IN, USA), CK-MB were also routinely evaluated. Neutrophil lymphocyte 
ratio (NLR) was calculated from routine blood analysis results. To detect the apical four-chamber view and measure left ventricular ejection fraction (LVEF), all patients underwent echocardiography within $48 \mathrm{~h}$ after PCI on the Vivid 7 ultrasound system (GE Medical Systems, USA).

\section{Quantitative real-time PCR:}

Total RNA was extracted from PBMC using TRIzol reagent (Invitrogen, Carlsbad, CA) according to the manufacturer's instructions. After quantification, cDNA was synthesised from $1 \mu \mathrm{g}$ of RNA using the FastQuant RT Kit First-Strand Synthesis Kit (TIANGEN, China). The reaction was performed at $42^{\circ}$ for $15 \mathrm{~min}$ and $95^{\circ}$ for $3 \mathrm{~min}$. RT-qPCR reactions were performed using SYBR Green master mix (Applied Biosystems, USA) with $\beta$-actin as an internal reference. All RTPCR reactions were performed in 96-well plates. Each sample was quantified twice for each lncRNA and the average was calculated. $\beta$-actin was selected as an internal reference. The RT PCR instrument used was ABI Prism 7900 (Applied Biosystems, USA). Shanghai Biological Engineering Co., Ltd synthesized the primers. The length of the primer is $18 \sim 29 \mathrm{bp}$; the length of the amplified product is between $250 \sim 350 \mathrm{bp}$; the primer is designed to span the intron region; the annealing temperature was 57 to $70^{\circ}$. The specific primer sequences are shown in Table 1 . The relative expression level of candidate lncRNA was calculated using the $2-^{\Delta \Delta \mathrm{CT}}$ Eqn.

\section{Follow-up and study endpoints:}

All patients were followed for $12 \pm 1$ mo. During this period, professionals continuously observed patients for the occurrence of HF, angina pectoris, recurrent infarction, readmission, and death. Followup mainly included outpatient visits and telephone calls. Information of patients was obtained from hospital records or phone contact with relatives of the patients. Recurrent angina readmission, HF, repeated stent intervention, gastrointestinal bleeding and outof-hospital death were defined as major adverse cardiovascular events (MACE).

\section{Cardiovascular risk factors:}

Body mass index (BMI) was calculated by dividing body weight $(\mathrm{kg})$ by height $\left(\mathrm{m}^{2}\right)$. Overweight/obesity was defined as a BMI $>=25$. People who reported regular tobacco use in the previous 6 mo were considered current smokers. Hypertension was defined as a history of hypertension and/or repeated systemic blood pressure (BP) measurements exceeding 140/90 $\mathrm{mmHg}$. Diabetes was defined as a history of the condition or the presence of diabetes and/or a fasting plasma glucose level $>7.0 \mathrm{mM}(126 \mathrm{mg} / \mathrm{dl})$ on two separate occasions, or a random glucose value $>11.1 \mathrm{mM}$ $(200 \mathrm{mg} / \mathrm{dl})$ on at least one occasion before the present admission. Concentrations of TC $>6.22 \mathrm{mM}$, TG $>$ $2.26 \mathrm{mM}$, LDL-C $>4.14 \mathrm{mM}$, and HDL-C $<1.04 \mathrm{mM}$ were defined as hypercholesterolemia, hypertriglyceridemia, high LDL-C, and low HDL-C, respectively, according to Chinese dyslipidemia guidelines. Dyslipidemia was defined as any of the 4 lipid abnormalities above.

\section{Statistical analysis:}

The data were analyzed using SPSS version 19.0 (IBM Corp. Released 2011. IBM SPSS Statistics for Windows, Version 20.0. Armonk, NY, USA: IBM Corp) software and GraphPad Prism 6.0 (GraphPad Software, San Diego, CA, USA; www.graphpad.com). Continuous variables with a Gaussian distribution are presented as mean \pm standard deviation (SD), and those with a non-Gaussian distribution are presented as median values with the corresponding 25 th to 75 th percentiles. Non-normal distributions are represented as Median and interquartile range (25th to 75 th percentiles Comparisons). Comparison between two groups was performed with Student's t-test or the Mann-Whitney $\mathrm{U}$ test. For comparisons among more than 2 groups, one-way ANOVA or the Kruskal Wallis test was used, as appropriate. The Pearson $\chi^{2}$ test was employed to compare qualitativevariables represented as frequencies. Correlation between 2 variables was analysed using Pearson correlation analysis or Spearman rank correlation. ROC curves were constructed to evaluate the predictive power of IncRNA in PBMCs between the MACE and non-MACE patients, and area under the curve (AUC) was used to assess the diagnostic value

\section{TABLE 1: PRIMERS USED FOR QRT-PCR}

\begin{tabular}{|c|c|c|}
\hline Name & & Sequence \\
\hline \multirow{2}{*}{ B-actin } & Forward & CTCCATCCTGGCCTCGCTGT \\
\hline & Reverse & GCTGTCACCTTCACCGTT-CC \\
\hline \multirow{2}{*}{ ENST00000581794.1 } & Forward & CCCATTGGAGAGGACTGTGG \\
\hline & Reverse & AGGGATTTTCAGCCAAGGGTA \\
\hline
\end{tabular}


of lncRNA. Sensitivity and specificity were calculated according to the standard formulas. Multivariable Cox regression analysis also was carried out to evaluate the predictive value of lncRNA for MACE. P values $<0.05$ were considered to indicate statistical significance in all comparisons.

\section{RESULTS AND DISCUSSION}

Baseline clinical characteristics of the study population are shown in Table 2. In the study, 58 AMI patients and 58 healthy controls were examined to determine the correlation of ENST00000581794.1 with AMI. Levels of ENST00000581794.1 in PBMCs were higher in AMI patients than in the control group (fig. 1). Patients with AMI showed higher rates of hypertension $(\mathrm{p}<0.001)$, diabetes mellitus $(\mathrm{p}=0.015)$, smoking $(\mathrm{p}<0.001)$, higher WBC counts $(p<0.001)$, NLR $(p<0.001)$, LDL-C $(p=0.012)$ and CK-MB $(p<0.001)$ and lower LVEF $(p<0.001)$. There were no differences in age, sex, cholesterol, blood pressure, or heart rate between the AMI group and the control group.

Rhe inflammatory markers WBC, NLR and the classic AMI biomarkers CK-MB, cTnI, were selected to analyze possible correlation with ENST00000581794.1 level. Correlation analysis results showed that ENST00000581794.1 was positively correlated with NLR $(\mathrm{r}=0.299, \mathrm{p}=0.023)$ and CK-MB $\quad(\mathrm{r}=0.302$, $\mathrm{p}=0.021$, Table 3).

Having established that ENST00000581794.1 in PBMCs is a risk factor for AMI, the predictive effect of ENST00000581794.1 on MACE in patients with AMI was explored. A total of 42 non-MACE patients and 16 MACE patients were studied. Of these patients, 9 were hospitalized owing to recurrent angina during follow-up, and 2 patients were hospitalized because of HF. Besides, 3 patients underwent re-stent implantation, one patient had gastrointestinal bleeding, and one experienced cardiac death. Patients with MACE showed higher CK-MB $(\mathrm{p}=0.019)$. The result of $\mathrm{cTnI}(\mathrm{p}=0.054)$ could be related to the smaller sample size. There were no statistical differences in age, sex, HDL, LDL, LVEF, history of diabetes, or smoking status between the MACE and non-MACE participants (Table 4). ROC analysis was performed to evaluate the predictive power of ENST00000581794.1 in PBMCs for MACE. The AUC was 0.744 (95\% CI: 0.61 0.88, $\mathrm{p}=0.004$ ) for ENST00000581794.1 (fig. 2).

When the Youden index was the largest, the cutoff value is 3.032. According to the cut-off value,

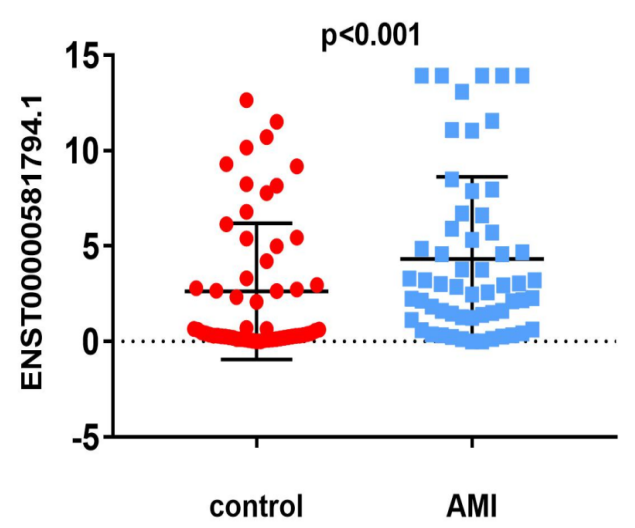

Fig. 1: Expression of ENST00000581794.1 in the two groups

TABLE 2: BASELINE CHARACTERISTICS OF STUDY PARTICIPANTS

\begin{tabular}{|c|c|c|c|}
\hline Variables & Control $(n=58)$ & AMI $(n=58)$ & p-value \\
\hline Age (y) & $54 \pm 9$ & $55 \pm 10$ & 0.750 \\
\hline Male, n (\%) & $30(51.7)$ & $39(67.2)$ & 0.089 \\
\hline Hypertension, n (\%) & $2(3.5)$ & $34(58.6)$ & $<0.001$ \\
\hline Diabetes mellitus, $\mathrm{n}(\%)$ & $1(1.7)$ & $8(13.8)$ & 0.015 \\
\hline Current smoker, n (\%) & $12(20.7)$ & $29(50)$ & $<0.001$ \\
\hline $\mathrm{SBP}(\mathrm{mmHg})$ & $118 \pm 18$ & $122 \pm 29$ & 0.322 \\
\hline $\mathrm{DBP}(\mathrm{mmHg})$ & $73 \pm 12$ & $77 \pm 13$ & 0.082 \\
\hline Heart rate (beats/min) & $77 \pm 12$ & $81 \pm 13$ & 0.139 \\
\hline WBC $\left(10^{9} / \mathrm{l}\right)$ & $6.31 \pm 1.59$ & $9.64 \pm 3.03$ & $<0.001$ \\
\hline NLR (n, \%) & $2.02 \pm 1.29$ & $6.42 \pm 5.36$ & $<0.001$ \\
\hline $\mathrm{TC}(\mathrm{mM})$ & $4.02 \pm 0.85$ & $4.39 \pm 1.17$ & 0.059 \\
\hline $\mathrm{TG}(\mathrm{mM})$ & $1.75 \pm 1.06$ & $1.67 \pm 1.10$ & 0.686 \\
\hline LDL-C (mM) & $2.56 \pm 0.73$ & $2.99 \pm 1.01$ & 0.012 \\
\hline CK-MB (U/L) & $13.94(11.20 \sim 17)$ & $26.8(16.1 \sim 60.61)$ & $<0.001$ \\
\hline LVEF (\%) & $63 \pm 5$ & $58 \pm 6$ & $<0.001$ \\
\hline ENST00000581794.1 & $0.52(0.22 \sim 4.21)$ & $2.9(1.27 \sim 5.92)$ & $<0.001$ \\
\hline
\end{tabular}

SBP is systolic blood pressure, DBP is diastolic blood pressure, WBC- white blood cells, NLR is neutrophil lymphocyte ratio, TC- serum total cholesterol, TG- triglyceride, LDL-C- low density lipoprotein, CK-MB- creatine kinase-MB, LVEF- left ventricular ejection fractions, Data are presented as the Mean $\pm S D$, median (interquartile range) or \%. $\mathrm{p}<0.05$ indicated statistically significant difference between groups 
patients with different expression levels divided into high $(n=27)$ and low expression groups $(n=31)$. The sensitivity and specificity at the optimal cut-off were 81.25 and $66.67 \%$, respectively. Log-rank tests of the 2 groups of survival curves were statistically significant $(p=0.001)$. The incidence of total MACE in the highexpression group was significantly greater than that in the low-expression group (fig. 3), as were the incidences of recurrent angina, HF, stent reimplantation, gastrointestinal bleeding, and cardiac death. Survival curve analysis showed that the incidence of MACE became significantly higher in patients who had high expression of ENST00000581794.1 as the observation time increased.

Multivariate analysis by stepwise Cox proportional hazards regression analysis (incorporating as putative predictors including age, gender, hypertension,

TABLE 3: CORRELATION ANALYSIS

\begin{tabular}{lcc}
\hline ENST00000581794.1 & $\mathbf{r}$ & $\mathbf{P}$ \\
\hline WBC, $\left(10^{9} / \mathrm{l}\right)$ & 0.056 & 0.674 \\
NLR, $\mathrm{n}(\%)$ & 0.299 & 0.023 \\
CK-MB, $(\mathrm{U} / \mathrm{l})$ & 0.302 & 0.021 \\
CTnl, $(\mu \mathrm{g} / \mathrm{l})$ & 0.083 & 0.544
\end{tabular}

WBC- white blood cells, NLR is neutrophil-lymphocyte ratio, CK-MBcreatine kinase-MB and $c T n l-$ cardiac troponin. $p<0.05$ indicated statistically significant difference between groups current smoker, CK-MB, cTnI, GRACE, Killip score, ENST00000581794.1) confirmed that CKMB (HR=1.01, $95 \%$ CI:1.00 1.01, p=0.013), cTnI (HR=1.07, $95 \% \mathrm{CI}: 1.02 \sim 1.13, \mathrm{p}=0.008)$, GRACE $(\mathrm{HR}=1.04,95 \% \quad \mathrm{CI}: 1.01 \sim 1.07, \quad \mathrm{p}=0.013) \quad$ and ENST00000581794.1 (HR $=1.17,95 \%$ CI: $1.00 \sim 1.37$,

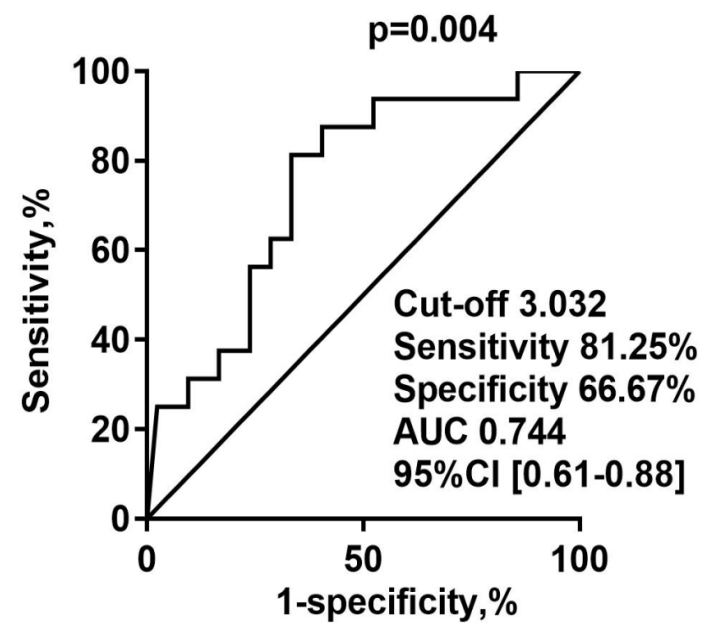

Fig. 2: ROC curve for MACE

Receiver operator characteristic (ROC) curve for the major adverse cardiovascular events (MACE). The area under the curve is 0.744 , and $95 \%$ confidence interval is 0.61-0.88

TABLE 4: COMPARISON OF CHARACTERISTICS AND BIOMARKERS AT ADMISSION BETWEEN MACE AND NON-MACE GROUP DURING THE FOLLOW-UP PERIOD

\begin{tabular}{|c|c|c|c|}
\hline Variables & Non-MACE $(n=42)$ & MACE $(n=16)$ & $\mathrm{p}$-value \\
\hline Age $(y)$ & $55 \pm 9$ & $55 \pm 13$ & 0.981 \\
\hline Male, n (\%) & $30(71.4)$ & $9(56.3)$ & 0.271 \\
\hline Hypertension, n (\%) & $20(47.6)$ & $14(87.5)$ & 0.006 \\
\hline Diabetes mellitus, n (\%) & $6(14.3)$ & $2(12.5)$ & 0.860 \\
\hline Current smoker, n (\%) & $21(50)$ & $8(50)$ & 1 \\
\hline SBP (mmHg) & $124 \pm 27$ & $118 \pm 36$ & 0.472 \\
\hline $\mathrm{DBP}(\mathrm{mmHg})$ & $77 \pm 13$ & $77 \pm 15$ & 0.872 \\
\hline Heart rate (beats/min) & $80 \pm 13$ & $82 \pm 11$ & 0.702 \\
\hline WBC $\left(10^{9} / L\right)$ & $9.53 \pm 3.01$ & $9.94 \pm 3.18$ & 0.652 \\
\hline NLR, n (\%) & $5.86 \pm 5.21$ & $7.89 \pm 7.89$ & 0.201 \\
\hline TC (mmol/L) & $4.40 \pm 1.14$ & $4.34 \pm 1.27$ & 0.856 \\
\hline TG (mmol/L) & $1.83 \pm 1.25$ & $1.25 \pm 0.36$ & 0.072 \\
\hline LDL-C (mmol/l) & $3.03 \pm 0.98$ & $2.87 \pm 1.11$ & 0.593 \\
\hline CK-MB (U/L) & $100.43(55.74 \sim 128)$ & 169 (80 424.5) & 0.019 \\
\hline$c T n l(\mu g / L)$ & $3.74 \pm 8.51$ & $9.78 \pm 13.87$ & 0.054 \\
\hline LVEF (\%) & $57.9 \pm 6.69$ & $58.69 \pm 5.36$ & 0.677 \\
\hline Killip score & $2.5(1)$ & $3(1)$ & 0.685 \\
\hline GRACE score & $121.14 \pm 32.75$ & $129.37 \pm 33.39$ & 0.398 \\
\hline Gensini score & $73.81 \pm 44.17$ & $66.31 \pm 29.21$ & 0.533 \\
\hline VTE score & $4.98 \pm 1.32$ & $4.43 \pm 1.55$ & 0.189 \\
\hline ENST00000581794.1 & $2.15(0.63 \sim 4.58)$ & $4.76(3.13 \sim 12.48)$ & $<0.001$ \\
\hline
\end{tabular}

SBP is systolic blood pressure, DBP is diastolic blood pressure, WBC- white blood cells, NLR is neutrophil lymphocyte ratio, TC- serum total cholesterol, TG- triglyceride, LDL-C- low density lipoprotein, CK-MB- creatine kinase-MB, LVEF- left ventricular ejection fractions. Data are presented as the Mean $\pm S D$, median (interquartile range) or \%. $p<0.05$ indicated statistically significant difference between groups 


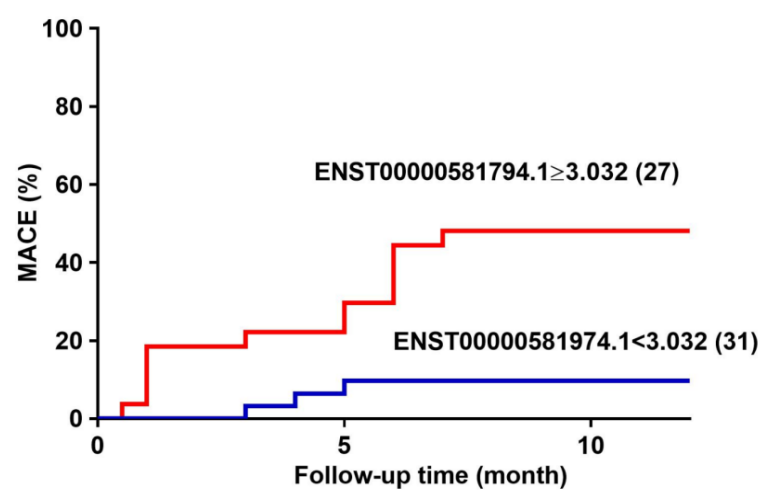

Fig. 3: Survival curve analysis of AMI patients with MACE

Survival curve analysis of acute myocardial infarction (AMI) patients with major adverse cardiovascular events (MACE). The incidence of MACE was significantly higher in the high expression group than in the low expression group $(p=0.001)$

TABLE 5: MULTIVARIATE COX REGRESSION ANALYSIS OF MACE

\begin{tabular}{lcc}
\hline Parameters & HR $(95 \% \mathrm{Cl})$ & p-value \\
\hline Age $(\mathrm{y})$ & $0.95(0.91 \sim 1.00)$ & 0.047 \\
Gender, n (\%) & $0.78(0.76 \sim 8.05)$ & 0.837 \\
Hypertension, n (\%) & $0.02(0.01 \sim 0.47)$ & 0.016 \\
Current smoker, n (\%) & $0.47(0.45 \sim 4.88)$ & 0.524 \\
CK-MB, (U/l) & $1.01(1.00 \sim 1.01)$ & 0.013 \\
CTnl, $(\mu \mathrm{g} / \mathrm{l})$ & $1.07(1.02 \sim 1.13)$ & 0.008 \\
Killip score & $0.33(0.98 \sim 1.10)$ & 0.071 \\
GRACE score & $1.04(1.01 \sim 1.07)$ & 0.013 \\
ENST00000581794.1 & $1.17(1.00 \sim 1.37)$ & 0.048 \\
\hline
\end{tabular}

CK-MB- creatine kinase-MB and CTnl- cardiac troponin, Killip score- clinical classification of heart failure caused by acute myocardial infarction; GRACE score, the Global Registry of Acute Coronary Events risk score. $\mathrm{p}<0.05$ indicated statistically significant difference between groups

$\mathrm{p}=0.048$ ) were associated with an increased risk of $1 \mathrm{y}$ MACE in AMI patients (Table 5).

AMI is a serious cardiovascular event with high morbidity and mortality. Coronary occlusion and plaque rupture caused by atherosclerosis is the most common causes of AMI. With the development of social economy and changed lifestyle, AMI has become a serious worldwide cardiovascular disease ${ }^{[13]}$. In recent years, the mortality rate of AMI has been rising in China. AMI is a complex, multifactorial disease that is the result of interactions between genetics and environmental factors. IncRNAs were shown to modulate gene expression at the epigenetic, transcriptional, and posttranscriptional levels ${ }^{[14,15]}$. Several studies have revealed dysregulation of IncRNAs in various disease states, including cancer, highlighting lncRNAs' potential clinical applications as diagnostic and prognostic biomarkers or therapeutic targets. lncRNA acts as a modulator of protein expression. It is thought not only to function in the development of organisms but also to play roles in various physiological and pathological conditions, including AMI ${ }^{[16]}$. Early and timely AMI diagnosis can prompt immediate reperfusion therapy, which greatly reduces mortality. Recent studies showed that MI is closely related to altered lncRNA expression, highlighting the potential of lncRNA as a biomarker for the early diagnosis of MI. However, the predictive value of IncRNA for the prognosis of MI had not been systematically studied.

To investigate whether ENST00000581794.1 could be a novel biomarker for the diagnosis of AMI, AMI patients and control groups were compared. With age and gender matching, the general risk factors for cardiovascular disease (smoking, hypertension, diabetes, and dyslipidemia) were significantly elevated in the AMI group. Compared to the control group, ENST00000581794.1 expression and CK-MB activity were significantly increased in patients with AMI. Moreover, Correlation analysis in AMI patients showed that ENST00000581794.1 had the same trend as CKMB.

Higher CK-MB in patients with myocardial infarction significantly increased the incidence and mortality of adverse cardiovascular events, and persistently high levels of CK-MB often indicated a poor prognosis. After a 12 mo follow-up, AMI patients with MACE had higher levels of CK-MB. Patients in the MACE group had higher ENST00000581794.1 expression than did patients in the non-MACE group. Similar to CK-MB, ENST00000581794.1 level may be useful in assessing the severity of myocardial damage in AMI patients. ENST00000581794.1 showed the optimal sensitivity and specificity for the diagnosis of MACE after AMI in the ROC curve analysis $(\mathrm{AUC}=0.744$, $95 \%$ CI: 0.61 0.88, p=0.004). Higher expression of the ENST00000581794.1 gene may increase the chances of poor prognosis in patients with AMI. Survival curve showed higher lncRNA expression level significantly increased risk of MACE compared with a low lncRNA expression level. These results showed that ENST00000581794.1 has a predictive value for the prognosis of AMI patients.

During AMI, excessive inflammation is a critical factor in aggravating cardiomyocyte injury and death. During AMI, acute myocardial necrosis and damaged matrix release endogenous alarm signals referred to as damage-associated molecular patterns (DAMPS). 
The release of DAMPs induces a pro-inflammatory response that mediates cardiomyocyte death through toll-like receptors (TLRs) and the recruitment of leukocytes into the infarct zone ${ }^{[17]}$. AMI is also associated with bone marrow activation via sympathetic nervous system activation and spleen monocytopoiesis, resulting in increased leukocyte influx, which in turn, aggravates atherosclerosis and contributes to recurrent $\mathrm{MI}^{[18]}$. Studies have found that neutrophils mediate plaque rupture and thrombosis by secreting proteolytic enzymes to cause vascular injury, activate the coagulation pathway and secrete pro-inflammatory cytokines $^{[19]}$, Lymphocytes represent the immune system regulatory pathway. During the occurrence of coronary heart disease, physiological stress and activation of the neurohormonal system cause cortisol release to mediate lymphopenia ${ }^{[20]}$.

Recent findings have determined that several lncRNAs act as inflammatory biomarkers after AMI. Previous reports have showed that ANRIL is positively associated with the percentage of lymphocytes and monocytes but inversely associated with white blood cell count, neutrophils, and platelets, MIAT is positively associated with lymphocyte count; and hypoxiainducible factor $1 \alpha$ antisense RNA2 is positively associated with white blood cell count, neutrophil count, and C-reactive protein ${ }^{[21]}$. In the current study, there were significant differences in WBC counts and NLR between AMI and control subjects. Correlation analysis showed that AMI patients had high expression levels of ENST00000581794.1 and higher NLR level. NLR is a novel inflammatory marker, which was shown by numerous clinical studies as an important guiding factor for the early diagnosis, condition evaluation and prognosis of coronary heart disease ${ }^{[22,23]}$. In summary, ENST00000581794.1 could be considered to cause myocardial injury by promoting inflammatory cell infiltration. Besides, through the co-expression network diagram, it was found that ENST00000581794.1 is closely related to 3 genes, especially GNB5, which could participate in arrhythmia through the potassium channel activated by acetylcholine, so this might provide a mechanism for the occurrence of arrhythmia after myocardial infarction. The co-expression network diagram is shown in fig. 4 .

Cox regression analysis revealed that CK-MB, cTnI, GRACE score and ENST00000581794.1 were independent predictors of MACE after AMI. This study found that ENST00000581794.1 could serve as a potential biomarker for AMI and could predict AMI severity and progression. It should be cautioned that lncRNA is highly expressed in many diseases, including cancers and maybe a predictive biomarker for these conditions as well. Therefore, when lncRNA is used as a biomarker for AMI, these other conditions must be excluded. The application of detection technology to lncRNA is challenging. Although RT-qPCR is the gold standard for quantitative gene expression and is considered a powerful technique for comparative expression analysis in the life sciences and medicine, it is very expensive and time-consuming for large numbers of samples. Finally, the importance of circulating lncRNAs in disease diagnosis is not well known. For example, it is unclear whether lncRNA causes disease or causes changes due to the disease itself.

This study found that ENST00000581794.1 is differentially expressed between AMI and the control group, which might serve as a potential biomarker for AMI and can predict the progression of AMI. However, this study has some limitations. Firstly, this study is a single-centre, small-sample study. Larger sample size is needed to further confirm the potential application of ENST00000581794.1 as a diagnostic tool for AMI. Secondly, it remains to be studied whether the dynamic monitoring of ENST00000581794.1 after admission has a higher predictive value for the prognosis of AMI. Although the diagnostic and predictive effects of ENST00000581794.1 on AMI in peripheral blood mononuclear cells was established, further research is needed to determine the consistency of ENST00000581794.1 expression in tissues and plasma. The clinical application value and expression mechanism of ENST00000581794.1 also needs to be investigated further. The elevated levels of expressed ENST00000581794.1 in PBMCs appear to be associated with increased risk of AMI and ENST00000581794.1 could serve as a promising new biomarker for the diagnosis and prognosis of AMI.

\section{Funding sources:}

This work was supported by a project grant from the Major Disease Medical Key Laboratory Open Subject of Xinjiang in China (2018D04029).

\section{Conflict of interest:}

All authors report no conflicts of interest in this work.

\section{REFERENCES}

1. Gabriel S, James SK, Atar D, Badano LP, Blömstrom-Lundqvist $\mathrm{C}$, Borger MA, et al. ESC Guidelines for the management of acute myocardial infarction in patients presenting with ST- 
segment elevation The Task Force on the management of STsegment elevation acute myocardial infarction of the European Society of Cardiology (ESC). Eur Heart J 2012;31: 2569-619.

2. Boersma E, Mercado N, Poldermans D, Gardien M, Vos J, Simoons ML. Acute myocardial infarction. Lancet 2003;361:847-58.

3. Li C, Pei F, Zhu X, Duan DD, Zeng C. Circulating microRNAs as novel and sensitive biomarkers of acute myocardial Infarction. Clin Biochem 2012;45:727-32.

4. Quan M, Chen J, Zhang D. Exploring the Secrets of Long Noncoding RNAs. Int J Mol Sci 2015;16:5467-96.

5. Li X, Wu Z, Fu X, Han W. IncRNAs: Insights into their function and mechanics in underlying disorders. Mutat ResRev Mutat 2014;762:1-21.

6. Kazemzadeh M, Safaralizadeh R, Orang AV. LncRNAs: emerging players in gene regulation and disease pathogenesis. J Genet 2015;94:771-84.

7. Archer K, Broskova Z, Bayoumi AS, Teoh JP, Davila A, Tang $\mathrm{Y}$, et al. Long Non-Coding RNAs as Master Regulators in Cardiovascular Diseases. Int J Mol Sci 2015;16:23651-67.

8. Ishii N, Ozaki K, Sato H, Mizuno H, Saito S, Takahashi A, et al. Identification of a novel non-coding RNA, MIAT, that confers risk of myocardial infarction. J Hum Genet 2006;51:1087-99.

9. Cheng J, Cai MY, Chen YN, Li ZC, Tang SS, Yang XL, et al. Variants in ANRIL gene correlated with its expression contribute to myocardial infarction risk. Oncotarget 2017;8:12607.

10. Yan Y, Zhang B, Liu N, Qi C, Xiao Y, Tian X. Circulating Long Noncoding RNA UCA1 as a Novel Biomarker of Acute Myocardial Infarction. Biomed Res Int 2016;1:1-7.

11. Kumarswamy R, Bauters C, Volkmann I, Maury F, Fetisch J, Holzmann A, et al. Circulating Long Noncoding RNA, LIPCAR, Predicts Survival in Patients With Heart Failure. Circ Res 2014;114:1569-75.

12. Arslan F, Bongartz L, Ten Berg JM, Jukema JW, Appelman Y, Liem AH, et al. 2017 ESC guidelines for the management of acute myocardial infarction in patients presenting with STsegment elevation: comments from the Dutch ACS working group. Neth Heart J 2018;26:417-21.

13. Li H, Ge J. Cardiovascular diseases in China: Current status and future perspectives. Int J Cardiol Heart Vasc 2015;6:25-31.
14. Mercer TR, Dinger ME, Mattick JS. Long non-coding RNAs: insights into functions. Nat Rev Genet 2009;10:155-9.

15. Hombach S, Kretz M. Non-coding RNAs: Classification, Biology and Functioning. Adv Exp Med Biol 2016;937:3-17.

16. Zhang Y, Sun L, Xuan L, Pan Z, Li K, Liu S, et al. Reciprocal Changes of Circulating Long Non-Coding RNAs ZFAS1 and CDR1AS Predict Acute Myocardial Infarction. Sci Rep 2016;6:22384

17. Frangogiannis NG. The Immune System and the Remodeling Infarcted Heart: Cell Biological Insights and Therapeutic Opportunities. J Cardiovasc Pharm T 2013;63:185

18. Dutta P, Courties G, Wei Y, Leuschner F, Gorbatov R, Robbins $\mathrm{CS}$, et al. Myocardial infarction accelerates atherosclerosis. Nature 2012;487:325-9.

19. Sawant AC, Adhikari P, Narra SR, Srivatsa SS, Mills PK, Srivatsa SS. Neutrophil to lymphocyte ratio predicts short- and long-term mortality following revascularization therapy for ST elevation myocardial infarction. Cardiol J 2014; 21:500-8.

20. Onsrud M, Thorsby E. Influence of in vivo Hydrocortisone on Some Human Blood Lymphocyte Subpopulations: I. Effect on Natural Killer Cell Activity. Scand J Immunol 2006;13:573-9.

21. Vausort M, Wagner DR, Devaux Y. Long Noncoding RNAs in Patients with Acute Myocardial Infarction. Circ Res 2014;115:668-77.

22. Zhang GY, Chen M, Yu ZM, Wang XD, Wang ZQ. Relation between neutrophil-to-lymphocyte ratio and severity of coronary artery stenosis. GMR 2014;13:9382-9.

23. Chen C, Cong BL, Wang M, Abdullah M, Wang XL, Zhang $\mathrm{YH}$, et al. Neutrophil to lymphocyte ratio as a predictor of myocardial damage and cardiac dysfunction in acute coronary syndrome patients. Integr Med Res 2018;S2213422018300416.

This is an open access article distributed under the terms of the Creative Commons Attribution-NonCommercial-ShareAlike 3.0 License, which allows others to remix, tweak, and build upon the work non-commercially, as long as the author is credited and the new creations are licensed under the identical terms

This article was originally published in a special
issue, "Biomedical Research in Healthcare Setting"
Indian J Pharm Sci 2020:82(2)Spl issue5;48-55

